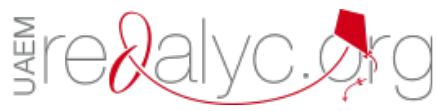

Centro Sur

ISSN: $2600-5743$

compasacademico@icloud.com

Grupo Compás

Ecuador

\title{
Artefactos pedagógicos: una experiencia innovadora
}

Silva Rodríguez, Yeimy Milena; Velásquez Camacho, Leida Yamile

Artefactos pedagógicos: una experiencia innovadora

Centro Sur, vol. 3, núm. 1, 2019

Grupo Compás, Ecuador

Disponible en: http://www.redalyc.org/articulo.oa?id=588861690009

Esta obra está bajo una Licencia Creative Commons Atribución-NoComercial-SinDerivar 4.0 Internacional. 


\title{
Artefactos pedagógicos: una experiencia innovadora
}

\author{
Pedagogical artifacts: an innovative experience \\ Yeimy Milena Silva Rodríguez \\ yeimy.silva@uniminuto.edu.co \\ Corporación Universitaria Minuto de Dios, Colombia \\ (D) http://orcid.org/0000-0003-1673-4693 \\ Leida Yamile Velásquez Camacho \\ lvelasque65@uniminuto.edu.co \\ Corporación Universitaria Minuto de Dios, Ecuador \\ http://orcid.org/0000-0002-0110-056X
}

Centro Sur, vol. 3, núm. 1, 2019

Grupo Compás, Ecuador

Recepción: 17 Junio 2018

Aprobación: 12 Febrero 2019

Redalyc: http://www.redalyc.org/ articulo.oa?id=588861690009
Resumen: El artefacto pedagógico es una herramienta educativa que busca fortalecer el proceso formativo de la población infantil de los Municipios que hacen parte de Sabana Centro específicamente en los campos de práctica profesional como: Instituciones Educativas, culturales, hospitalarias y fundaciones que trabajan en pro de los derechos y la formación integral de los mismos, por medio de la intervención pedagógica de los estudiantes de Lic. en Pedagogía Infantil de la modalidad presencial y distancia de la Corporación Universitaria Minuto de Dios UNIMINUTO. La finalidad de esta propuesta es elaborar artefactos pedagógicos a partir de las necesidades de los educandos, evidenciadas en los campos de práctica profesional identificando las necesidades de la población escolar por medio de actividades diagnósticas que permita evidenciar la dimensión del desarrollo infantil a fortalecer en el sitio de práctica. Este proceso busca planear, diseñar e implementar los artefactos pedagógicos en los campos de práctica, contrarrestando la problemática en el aula y así garantizar una educación exitosa.

Palabras clave: Práctica pedagógica, Artefacto Pedagógico, impacto social, dimensiones del desarrollo infantil, Innovación pedagógica.

Abstract: The pedagogical artifact is an educational tool that seeks to strengthen the educational process of the child population of the Municipalities that are part of Sabana Centro specifically in the fields of professional practice such as: Educational, cultural, hospital and foundations institutions that work for the rights and the integral formation of the same ones, by means of the pedagogical intervention of the students of Lic. in Pedagogía Infantil of the face-to-face modality and distance of the Corporación Universitaria Minuto de Dios UNIMINUTO. The purpose of this proposal is to develop pedagogical devices based on the needs of the learners, evidenced in the fields of professional practice, identifying the needs of the school population through diagnostic activities that show the dimension of child development to be strengthened on the site of practice. This process seeks to plan, design and implement pedagogical artifacts in the fields of practice, counteracting the problems in the classroom and thus ensure a successful education. Keyword: Pedagogical practice, Pedagogical artifact, social impact, dimensions of child development, pedagogical innovation. Introducción

Keywords: Pedagogical practice, Pedagogical artifact, social impact, dimensions of child development, pedagogical innovation.

\section{INTRODUCCIÓN}

Dentro de las nuevas propuestas educativas del contexto, nace la idea de crear material que motive a los estudiantes y a la vez apoye los procesos 
de formación de una manera integral, aportando de una manera didáctica a la solución de diferentes problemáticas que se presentan dentro de las aulas de clase. Es así, como se parte del ideal de no dar continuidad a una educación tradicional y poco lúdica donde el principal objetivo es el conocimiento dejando de lado las otras dimensiones del desarrollo. Lo anterior, da paso a la construcción de artefactos pedagógicos desde la práctica profesional donde las estudiantes de séptimo semestre del programa realizan un proceso de indagación y planeación donde buscan dar solución a una problemática central que descubren en el contexto a partir de un diagnostico introductorio.

Los estudiantes del programa realizan su acción pedagógica con niños y jóvenes quienes necesitan potenciar sus habilidades de una manera dinámica y significativa. Ausubel afirma que "toda experiencia que parte de los conocimientos y vivencias previas del sujeto -las mismas que son integradas con el nuevo conocimiento y se convierten en una experiencia significativa- se le conoce como aprendizaje significativo". Por lo anterior,

las estudiantes inician con un reconocimiento del contexto donde desarrollan actividades diagnosticas que permiten evidenciar conocimientos previos, fortalezas y aspectos a mejorar dentro de las dimensiones del desarrollo de los niños, donde se hace un análisis de los indicadores planteados acorde a la edad del grupo de trabajo. También permite evidenciar los estilos de aprendizaje y gustos de los estudiantes para direccionar la mejor metodología a llevar en la elaboración del artefacto.

Partiendo de lo planteado por Piaget, J (1896-1980) Afirma la reconfiguración de las estructuras cognitivas en base a la construcción constante de nuevos significados, dado que el protagonista de la enseñanza es el estudiante. Planteando cuatro etapas de desarrollo (sensorio motriz, pre operacional, operaciones concretas y formales)que permite tener en cuenta características propias de la edad con la que se va a trabajar.

Teniendo en cuenta lo anterior, se busca planear actividades enfocadas a las diferentes dimensiones como apoyo del artefacto. También las estudiantes van realizando registros de observación donde hacen un seguimiento al proceso de los niños, los avances logrados y refuerzos que deben hacer para cumplir con los objetivos propuestos.

Una intervención apropiada en los primeros años de vida ayuda a potenciar el desarrollo del cerebro, a través de experiencias vivenciales que fomenten la creatividad dentro del aula. Shore(1997), hace un análisis entre el viejo y nuevo pensamiento, donde hace hincapié en la relación que tienen las experiencias previas y las nuevas en relación al ambiente y las conexiones con el cerebro. Así mismo, Shore afirma que "el desarrollo del cerebro no es lineal: existen momentos claves para la adquisición de cada tipo de conocimiento y habilidades, donde el cerebro de los niños después de los tres años es doblemente activo". Lo anterior permite ver que es de gran importancia el impacto que se genere desde el contexto a través de la innovación, donde los niños son más perceptivos y logran potenciar habilidades en cada uno de ellos, vinculando las temáticas impuestas en 
los planes de estudio de las instituciones donde se realizan las prácticas profesionales.

Los artefactos pedagógicos que se elaboran durante las prácticas pedagógicas, vinculan los pilares de la educación, donde el juego tiene un papel importante en el desarrollo infantil. Es así, como María Carbonell (1987) aporta afirmando "que el niño ha de jugar precisamente, procuremos que juegue educándose o que se eduque jugando", contribuyendo positivamente al fin que se busca con la implementación de los artefactos en los diferentes campos de práctica. También se debe destacar la importancia que tiene el vincular los intereses particulares de los niños relacionados a sus vivencias del contexto, haciendo más atractivas las actividades a desarrollar dentro del artefacto. Manjon (1902) también aporta diciendo que "el niño es juguetón. Rebosa energías que se derraman en el juego, y de aquí se deduce una gran regla, que deja abierto el campo a múltiples pequeñas reglas que debe poner en juego la imaginación del maestro: enseñar jugando".

Contrastando lo antes mencionado con algunas investigaciones previas que se han realizado en relación a la construcción de material didáctico, es de gran relevancia destacar que estos artefactos parten de un diagnóstico previo, al cual buscan dar solución vinculando las diferentes dimensiones del desarrollo; es decir, no se da solución al problema si no que a la vez se está potenciando las diferentes dimensiones a partir de la problemática evidenciada.

Algunas investigaciones que se abordaron están relacionadas con la vinculación de material tecnológico en las aulas de clase, buscando dar solución a problemáticas en áreas específicas. Dos de estas fueron "Learning in the ocean", una investigación que parte de la necesidad de vincular un material tecnológico para la enseñanza de la suma y resta en primer y segundo año de primaria y Aprendo con Mily y Vily, material didáctico, herramienta que facilite procesos de enseñanza en la resolución pacífica de conflictos, cuya herramienta presentaba situaciones del contexto y crear nuevas soluciones a partir de videos digitales. Lo anterior deja ver la relevancia que tienen estos artefactos dentro de la formación preescolar, teniendo en cuenta que apuntan a una formación integral desde una problemática en particular.

\section{MATERIALES Y MÉTODOS}

Fue una investigación de carácter cualitativo debido a que "desarrolla procesos en términos descriptivos e interpreta acciones, lenguajes, hechos funcionalmente relevantes y los sitúa en una correlación con el más amplio contexto social”. (Martínez R. J. 2011), que "se orienta a profundizar casos específicos y no a generalizar. Su preocupación no es prioritariamente medir, sino cualificar y describir el fenómeno social a partir de rasgos determinantes"

Para Latorre (2003) "la investigación-acción es vista como una indagación práctica realizada por el profesorado, deforma colaborativa, con la finalidad de mejorar su práctica educativa a través de ciclos de 
acción y reflexión". Es decir una reflexión del quehacer pedagógico hacia la solución de problemas prácticos, buscando modificar una acción.

Dentro de este orden de ideas, se realizó una búsqueda selectiva de información en diferentes bases de datos, páginas web y libros de material teórico que aportara al tema de estudio, a partir del cual se buscó responder las preguntas de Investigación, cada una desde su temática central (línea de acción), para lograr tal fin, se llevó a cabo el siguiente procedimiento: una vez hecha la selección de información pertinente para cada línea, se pasó a revisar detalladamente cada documento, luego de un proceso de selección pertinente, se elaboraron 10 Resumes Analíticos en Educación (RAE), por cada línea de acción, posteriormente, se identificó una categoría de análisis especifica por línea, la cual fue estudiada mediante una Matriz de Análisis, a partir de cinco sub-categorías de análisis.

Así mismo se realiza con los estudiantes del programa una planeación y ejecución del artefacto pedagógico, llevando a cabo unas etapas que inician desde un diagnóstico, planeación de actividades, registros de ejecución, aplicación y sustentación.

Los estudiantes del programa diligencian un formato donde redactan su propuesta del Artefacto pedagógico que apoye el proceso de formación de los educandos, el cual contiene los siguientes requerimientos: nombre del artefacto, la temática de acuerdo al área, objetivo, dimensiones a trabajar: cognitiva, comunicativa, espiritual, estética, socio afectiva y/ o naturalista, descripción de actividades y evidencias fotográficas de la propuesta.

Posteriormente se aprueba por la Docente de Práctica Profesional e Investigación para dar el aval y construcción de la misma.

Estos artefactos pedagógicos se llevan realizando desde el año 2015, dando continuidad en el proceso durante 3 años con un número aproximado de 40 ejemplares por semestre académico, buscando patentes y convertirlos en una propuesta de negocio. Por consiguiente anexamos como evidencia unas de las muestras de artefactos pedagógicos innovadores:

1. Mimo más que una cara blanca: este artefacto pedagógico está elaborado para enseñanza del lenguaje de señas y contiene una serie de actividades que trabajaron como: asociación, fonemas, grafemas, partes del cuerpo, coordinación viso-manual, memoria, valores, auto reconocimiento. Comunicación y relaciones interpersonales. (Ver imagen N. 1)

2. El auto mágico: es artefacto pedagógico está enfocado a la dimensión cognitiva como: secuencias, asociación y nociones, integrando actividades que potencia las otras dimensiones con actividades como: coordinación viso-manual, estimulación sensorial y trabajo en grupo. (Ver imagen N. 2)

3. Factocional: Este artefacto pedagógico está enfocado a metodología de Pedagogía Conceptual de Miguel de Zubiria. A través de los mentefactos nocionales con actividades lúdico pedagógicas como las consonantes, auto-imagen, valores, estructura social y familiar, dialogo y cuidado ambiental. (Ver imagen N. 3) 
4. Fotunato: este artefacto pedagógico busca fortalecer la dimensión ética-moral a través de un cuento en 3D con imágenes que permiten ampliar el vocabulario y desarrollo de la imaginación. (Ver imagen N. 4)

5. Casita didáctica: este artefacto pedagógico se enfocó en estimulación, vinculando los cuatro pilares de la educación (Arte, juego, exploración del medio y literatura). (Ver imagen N. 5)

6. La casita mágica: este artefacto pedagógico trabajo las diferentes dimensiones con las siguientes temáticas: partes del cuerpo, valores, secuencias, conteo y trazo. (Ver imagen N. 6)

\section{RESULTADOS}

La elaboración de artefactos pedagógicos busca una relación entre los estudiantes de Licenciatura en Pedagogía Infantil y el impacto en la comunidad educativa de las instituciones educativas, hospitalarias, fundaciones y ludotecas de sabana centro, donde se identificó cada una de las necesidades que se viven comprendiendo que la sociedad juega un papel importante en el bienestar de los educandos. Por ello, las acciones y prácticas que se realizan con el artefacto pedagógico, permiten desarrollar un proceso de aprendizaje significativo, donde la comunidad debe hacer un paréntesis sobre cada una de las problemáticas que se evidenciaron en el contexto.

Por consiguiente el proceso que se realizó con el diagnóstico permitió evidenciar conocimientos previos, fortalezas y estilos de aprendizaje, para direccionar la metodología a llevar en la elaboración de los artefactos pedagógicos. Así mismo se buscó contrarrestar aspectos a fortalecer en las dimensiones del desarrollo de los niños, donde se hizo un

análisis de los indicadores, que direccionaron las actividades enfocadas a las diferentes dimensiones. Durante la estadía en cada una de las instituciones, las estudiantes también realizaron registros de observación donde hicieron un seguimiento al proceso de los niños, los avances logrados y refuerzos que debían hacer para cumplir con los objetivos propuestos.

\section{DISCUSIÓN}

También ejecutaron estrategias metodológicas que sirvieron para que los estudiantes del programa lograran identificar los problemas pedagógicos. Una estrategia, fue articular el juego como herramienta pedagógica, donde la comunidad se involucró buscando posibles soluciones a las problemáticas presentadas, generando un aprendizaje significativo articulando la imaginación y creatividad innovadora dando inicio a nuevos proyectos didácticos dentro de las instituciones.

Así mismo, se pudo evidenciar el impacto que causa la práctica de Licenciatura en Pedagogía Infantil de UNIMINUTO en Sabana Centro, generando nuevas propuestas de innovación pedagógica y buscando 
evento de apropiación social que permitan la divulgación del trabajo que se lleva realizando dentro del programa.

Finalmente, los artefactos pedagógicos son estrategias universales, transversales, humanas e interdisciplinares, que atiende de forma integral el cuidado y formación de los niños, desde la transformación de actitudes, la construcción de saberes, la articulación de experiencias y la planeación de propuestas que fortalezcan la relación hombre-medio.

Donde, el docente y demás actores sociales y educativos, son determinantes dentro de la selección, formulación, aplicación, adaptación y contextualización de estrategias pedagogías que desarrollen aprendizajes significativos y funcionales para el educando.

\section{Referencias}

Elliott, J. (1994). La investigación en la Educación. España: Morata, S.L.

Hernández Sampiere, R., Fernandez Collado, C., \& Baptista Lucio, M. (2003). Metodología de la Investigación. México: Mc Graw Hill.

Piaget, J. (1991). Seis Estudios de Psicología. Barcelona, España: Laboral. S.A.

Torres Carrillo, A. (1996). Estrategias y Técnicas de Investigación Cualitativa. Bogotá, Colombia: Guadalupe

Level, M.B, \& Mostacero, R. (2011). El texto escolar: ¿Artefacto didáctico? Investigación y Postgrado, 26(2), 9-59. Recuperado de http://www.scielo.org.ve/scielo.php?script=sci_arttext\&pid=S1316$00872011000200002 \& \operatorname{lng}=\mathrm{es} \& \mathrm{t} \operatorname{lng}=\mathrm{es}$.

Eming Young, Mary, \& Fujimoto-Gómez, Gaby. (2003). Desarrollo Infantil Temprano: lecciones de los programas no formales. Revista Latinoamericana de Ciencias Sociales, Niñez y Juventud, 1(1), 85-123. Recuperado de http://www.scielo.org.co/scielo.php?script=sci_arttext\&pid=S1692$715 \mathrm{X} 2003000100004 \& \operatorname{lng}=$ en\&tlng=es.

Rivera Muñoz, J. (2014).El aprendizaje significativo y la evaluación de los aprendizajes. Revista de investigación educativa. 8(14), 47-52. Recuperado de http://online.aliat.edu.mx/adistancia/dinamica/lecturas/ El_aprendizaje_significativo.pdf

Payà Rico, Andrés. (2014). Juego, juguete y educación en la pedagogía española contemporánea. Espacios en blanco. Serie indagaciones, 24(1), 107-126. Recuperado de https://www.redalyc.org/pdf/3845/384539806008.pdf 